\title{
Cavopulmonary assist in the neonate: An alternative strategy for single-ventricle palliation
}

\author{
Mark D. Rodefeld, MD \\ Jack H. Boyd, MDa \\ Cynthia D. Myers, MDa \\ Robert G. Presson, Jr, MD ${ }^{\mathrm{b}, \mathrm{c}}$ \\ Wiltz W. Wagner, Jr, PhD ${ }^{\mathrm{b}, \mathrm{c}}$ \\ John W. Brown, MD ${ }^{a}$
}

From the Department of Surgery, Section of Cardiothoracic Surgery, ${ }^{\mathrm{a}}$ and the Departments of Anesthesiology and Cellular and Integrative Physiology, ${ }^{\mathrm{C}}$ Indiana University School of Medicine and James Whitcomb Riley Hospital for Children, Indianapolis, Ind.

Read at the Twenty-ninth Annual Meeting of The Western Thoracic Surgical Association, Carlsbad, Calif, June 18-21, 2003.

Received for publication June 17, 2003; revisions requested Aug 22, 2003; revisions received Nov 5, 2003; accepted for publication Nov 12, 2003.

Address for reprints: Mark D. Rodefeld, MD, Department of Surgery, Section of Cardiothoracic Surgery, Indiana University School of Medicine, Emerson Hall 215, 545 Barnhill Dr, Indianapolis, IN 46202 (E-mail: rodefeld@iupui.edu).

J Thorac Cardiovasc Surg 2004;127:705-11 $0022-5223 / \$ 30.00$

Copyright (C) 2004 by The American Association for Thoracic Surgery

doi:10.1016/j.jtcvs.2003.11.007
Background: Cavopulmonary blood flow, rather than a systemic arterial source of pulmonary blood flow, stabilizes Norwood physiology. We hypothesized that pumpassisted cavopulmonary diversion would yield stable pulmonary and systemic hemodynamics in the neonate. This was tested in a newborn animal model of total cavopulmonary diversion and univentricular Fontan circulation.

Methods: Lambs $(\mathrm{n}=13$; mean weight, $5.6 \pm 1.5 \mathrm{~kg}$; mean age, $6.8 \pm 4.0$ days) were anesthetized and mechanically ventilated. Baseline hemodynamic parameters were measured. Total cavopulmonary diversion was performed with bicaval venous-to-main pulmonary artery cannulation. A miniature centrifugal pump was used to assist cavopulmonary flow. Support was titrated to normal physiologic parameters. Hemodynamic data, arterial blood gases, and lactate values were measured for 8 hours. Baseline, 1-hour, and 8-hour time points were compared by using analysis of variance.

Results: All animals remained stable without the use of volume loading, inotropic support, or pulmonary vasodilator therapy. Cardiac index, systemic arterial pressure, left atrial pressure, and lactate values were similar to baseline values 8 hours after surgery. Mean pulmonary arterial pressure and pulmonary vascular resistance were modestly increased 8 hours after surgery. Mean arterial $\mathrm{pH}, \mathrm{Po}_{2}$, and $\mathrm{PCO}_{2}$ values remained stable throughout the study.

Conclusions: Cavopulmonary assist is feasible in a neonatal animal model of total cavopulmonary diversion and univentricular Fontan circulation with acceptable pulmonary arterial pressures and without altering regional volume distribution or cardiac output. Pump-assisted cavopulmonary diversion, in combination with Norwood aortic arch reconstruction, could solve several major problems associated with a systemic shunt-dependent univentricular circulation, including hypoxemia, impaired diastolic coronary perfusion, and ventricular volume overload.

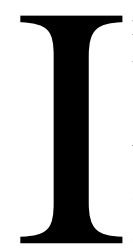
nstability and mortality remain significant problems for palliation of singleventricle cardiac anomalies. This is especially problematic for stage 1 surgical palliation of hypoplastic left heart syndrome, despite refinements in technique and clinical management. ${ }^{1,2}$ Interstage mortality, often sudden and unexplained, ${ }^{3,4}$ ranges from $4 \%$ to $24 \%$ and is a direct consequence of an unstable physiologic arrangement. A systemic source of pulmonary blood flow places the pulmonary and systemic circulations in parallel, and the single morphologically right ventricle must support twice the normal ventricular volume. Hypoxemia $\left(\mathrm{Po}_{2}\right.$ of approximately $\left.35-40 \mathrm{~mm} \mathrm{Hg}\right)$, which is mandatory to prevent pulmonary overcirculation, depresses myocardial and neurocognitive function, ${ }^{5}$ 
stimulates pulmonary hypertension, and prevents normal involution of the pulmonary arteriolar medial muscle layer. ${ }^{6}$

The leading identifiable causes of death after the Norwood operation are inadequate coronary blood flow and either excessive or inadequate pulmonary blood flow, all of which are directly linked to the systemic-to-pulmonary arterial shunt. ${ }^{7}$ Coronary perfusion, which normally occurs predominantly during diastole, shifts to a systolic-predominant pattern because of diastolic shunt runoff and ventricular volume overload and, as such, is further limited by increased myocardial wall tension. ${ }^{8}$ The recently described right ventricle-to-pulmonary artery shunt addresses the important concern of impaired diastolic coronary perfusion; however, it does not alleviate the problems of hypoxemia, parallel circulations, and volume loading of the ventricle, which may in fact be made worse because of conduit insufficiency. ${ }^{9}$

Synthetic shunts have inherent issues, including thrombosis and subjectivity in selecting shunt length and diameter. ${ }^{4}$ Consequently, suboptimal outcomes in stage 1 Norwood physiology are often attributable to shunt-related problems. In contrast, stability and survival improve dramatically in patients after stage 2 and stage 3 palliation of hypoplastic left heart syndrome, a direct corollary to takedown of the systemic shunt and establishment of cavopulmonary blood flow. ${ }^{2}$

A principal obstacle to combining elements of the second- or third-stage procedures (or both) with the first-stage operation is the potential for increased pulmonary vascular resistance in the neonate..$^{10}$ As currently practiced, a highpressure source of pulmonary blood flow, via a systemicto-pulmonary arterial shunt, is required to overcome potentially increased pulmonary vascular resistance. It stands to reason that the ability to eliminate the problematic systemic arterial shunt and instead provide a systemic venous source of pulmonary blood flow would be of enormous benefit. We hypothesized that pump-assisted cavopulmonary diversion would yield stable pulmonary and systemic hemodynamics and maintain pulmonary gas exchange function in the neonate without altering systemic venous volume distribution or cardiac output. This concept was tested in a newborn animal model of total cavopulmonary diversion and univentricular Fontan circulation.

\section{Methods}

The experimental protocol was approved by the Animal Care and Use Committee of the Indiana University School of Medicine. Animals received humane care in accordance with the Principles of Laboratory Animal Care formulated by the National Society for Medical Research and the Guide for the Care and Use of Laboratory Animals prepared by the Institute of Laboratory Animal Resources and published by the National Institutes of Health (National Institutes of Health publication 85-23, revised 1985).
Thirteen newborn lambs (mean weight, $5.6 \pm 1.5 \mathrm{~kg}$; mean age, 6.8 days; range, 3 to 15 days) underwent mask induction, followed by endotracheal intubation and mechanical ventilation with a Servo 900C volume-cycled respirator (Siemens, Danvers, Mass) with $100 \%$ oxygen and $0.7 \%$ to $1.5 \%$ isoflurane. Ventilation was maintained at 32 to 38 breaths per minute with tidal volumes of 12 to $15 \mathrm{~mL} / \mathrm{kg}$ and $4 \mathrm{~cm} \mathrm{H}_{2} \mathrm{O}$ positive end-expiratory pressure. Minor ventilator adjustments were made to maintain a $\mathrm{PCO}_{2}$ of approximately $35 \mathrm{~mm} \mathrm{Hg}$. Temperature was maintained at $38.5^{\circ} \mathrm{C}$ with a thermal pad.

A femoral arterial line (Intracath 16 gauge; Becton Dickinson, Sandy, Utah) was placed for systemic blood pressure monitoring. A femoral venous line was advanced to the infradiaphragmatic vena cava for measurement of systemic venous pressure. The heart was exposed through a median sternotomy, and the pericardium was suspended. The azygous vein and ductus arteriosus were ligated. Pressure-monitoring lines were placed in the left atrial appendage, distal main pulmonary artery, and proximal superior vena cava. An ultrasonic flowprobe (model 12A; Transonic Systems Inc, Ithaca, NY) was placed around the ascending aorta. Baseline systemic arterial pressure, pulmonary arterial pressure, left atrial pressure, vena caval pressure, and cardiac output were measured. Baseline activated clotting time, arterial blood gas, and lactate values were also obtained. Mean circulatory filling pressure, an indicator of unstressed systemic volume status, was measured in 5 animals by recording inferior vena caval pressure after 7 seconds of induced ventricular fibrillation. ${ }^{11}$

\section{Total Cavopulmonary Diversion}

After systemic heparinization (sodium heparin, $150 \mathrm{U} / \mathrm{kg}$ ), pursestring sutures were placed in the superior vena cava, inferior vena cava, proximal main pulmonary artery, and right atrial appendage. A decompression cannula (left heart vent catheter, 13F; Medtronic Inc, Minneapolis, Minn) was introduced through the right atrial appendage into the right ventricle for egress of thebesian and coronary venous blood. Bicaval venous (single-stage venous drainage cannula, $18 \mathrm{~F}$ [inferior vena cava] and 14F [superior vena cava]; Edwards Lifesciences, Irvine, Calif) and pulmonary arterial cannulation (pulmonary arterial cannula, 18F; A-Med Systems Inc, West Sacramento, Calif) was performed (Figure 1). The cannulas were connected to a miniature centrifugal pump (Paraflow; A-Med Systems Inc), and the circuit was deaired (volume, $45 \mathrm{~mL}$ ). The pump was activated, and flow was gradually increased over several minutes. Pump output was titrated to match baseline cardiac output. Caval occlusion tourniquets were tightened to produce inflow occlusion to the right heart, and a vascular clamp was placed across the proximal main pulmonary artery to prohibit right ventricular contribution to pulmonary blood flow.

\section{Fluid Management and Data Acquisition}

Intravenous fluids were administered to account for insensible loss in an open chest animal preparation after major surgical intervention. Additional fluids were administered if inferior vena caval pressure was lower than baseline or if impaired venous drainage due to intermittent obstruction was apparent by tugging on the venous cannulas or by a reduction in pump flow rate without a change in pump speed. Surgical blood loss was replaced 1:1 with fresh heparinized homologous blood. Activated clotting time was 


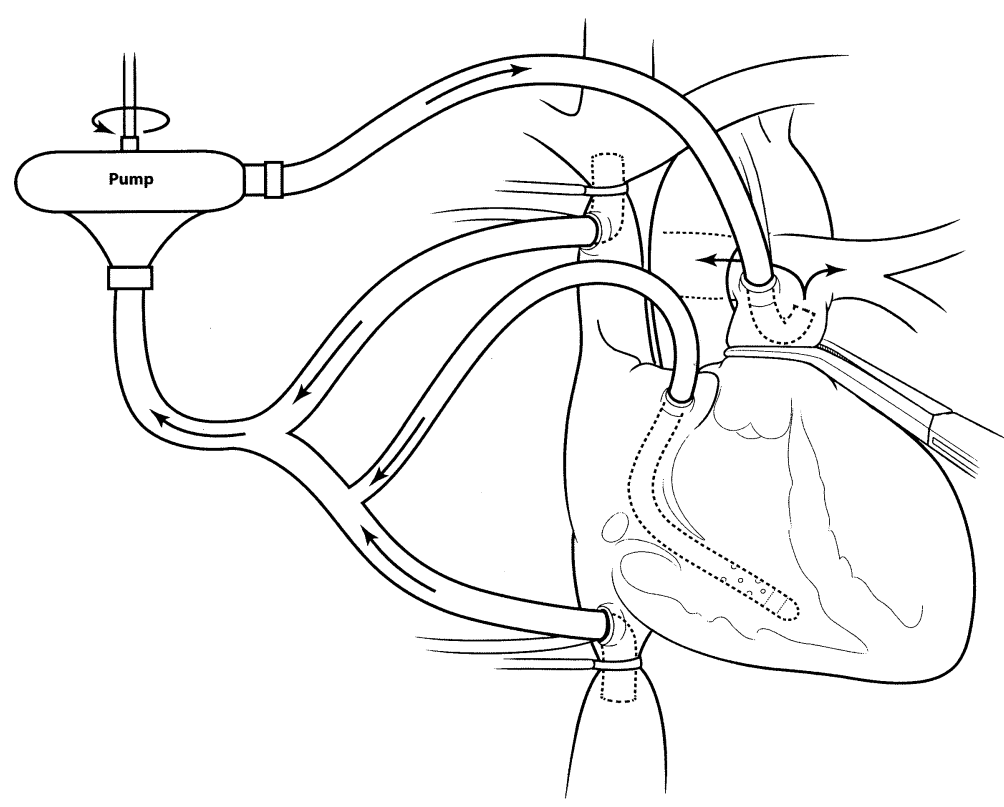

Figure 1. Total cavopulmonary diversion using bicaval venous-to-pulmonary artery cannulation. A miniature centrifugal pump was used to augment cavopulmonary flow. A decompression cannula was placed into the right ventricle to divert thebesian and coronary venous blood to the low-pressure systemic venous circulation. The proximal main pulmonary artery was clamped to exclude right ventricular contribution to pulmonary blood flow.

maintained greater than 1.5 times baseline. A surface electrocardiogram and systemic arterial waveform were continuously monitored (HP7354C physiologic monitor; Hewlett-Packard, Palo Alto, Calif). Hemoglobin was measured with an OSM3 hemoximeter (Radiometer, Westlake, Ohio) calibrated for measurement of sheep hemoglobin. Arterial blood gases, corrected for hemoglobin level, were measured with an ABL 500 blood gas analyzer (Radiometer). Arterial lactate values were measured with a YSI 2300 Stat Plus glucose and lactate analyzer (YSI, Yellow Springs, Ohio). Hemodynamic data and arterial blood gases were measured at hourly intervals for 8 hours after initiation of cavopulmonary assist. The postoperative mean circulatory filling pressure was measured at 8 hours $(n=5)$.

\section{Statistical Analysis}

Samples are reported as mean \pm SD. Comparison of baseline values with 1- and 8-hour time points after initiation of cavopulmonary assist was performed with analysis of variance. Statistical analysis was performed with SigmaStat software (SPSS Inc, Chicago, Ill).

\section{Results}

Hemodynamic and pulmonary function data are shown in Table 1. Cardiopulmonary function remained stable throughout the cavopulmonary assist period without modulation of pulmonary vascular tone, addition of inotropes, or excessive volume loading. Cardiac index and systolic blood pressure remained stable throughout the course of the study, but diastolic blood pressure decreased significantly. Systemic venous pressure and mean circulatory filling pressure increased only moderately from baseline conditions. This is an important difference that distinguishes our model from similar models of univentricular Fontan circulation (without mechanical assist) in which nonphysiologic volume loading was necessary to produce hemodynamic stability. ${ }^{12,13}$

At baseline, pulmonary arterial pressure was expressed as the mean of pulsatile flow. Because blood flow was nonpulsatile under cavopulmonary assist conditions, the absolute pressure was expressed. Pulmonary arterial pressures were modestly increased (mean, $23.3 \mathrm{~mm} \mathrm{Hg}$ at 8 hours of cavopulmonary assist) when compared with baseline (13.1 $\mathrm{mm} \mathrm{Hg}$ ), and severe pulmonary hypertensive events were not encountered. Pulmonary vascular resistance remained significantly increased from baseline throughout the cavopulmonary assist period. This value, however, showed a trend toward normalization and at 8 hours was significantly lower than values in the early cavopulmonary assist period (Figure 2).

Hemoglobin levels were significantly decreased at 8 hours even though fresh, heparinized whole blood was administered to replace measurable blood loss. The decline in serum hemoglobin was attributed to unquantified blood loss and a mild dilutional effect from crystalloid administration. The $\mathrm{pH}$ remained within the normal range despite a statistically significant decrease at 8 hours. Gas exchange function remained excellent in all cases. Ventilator adjustments did not deviate more than $10 \%$ from baseline settings, and peak airway pressures remained less than $25 \mathrm{~cm} \mathrm{H}_{2} \mathrm{O}$. 


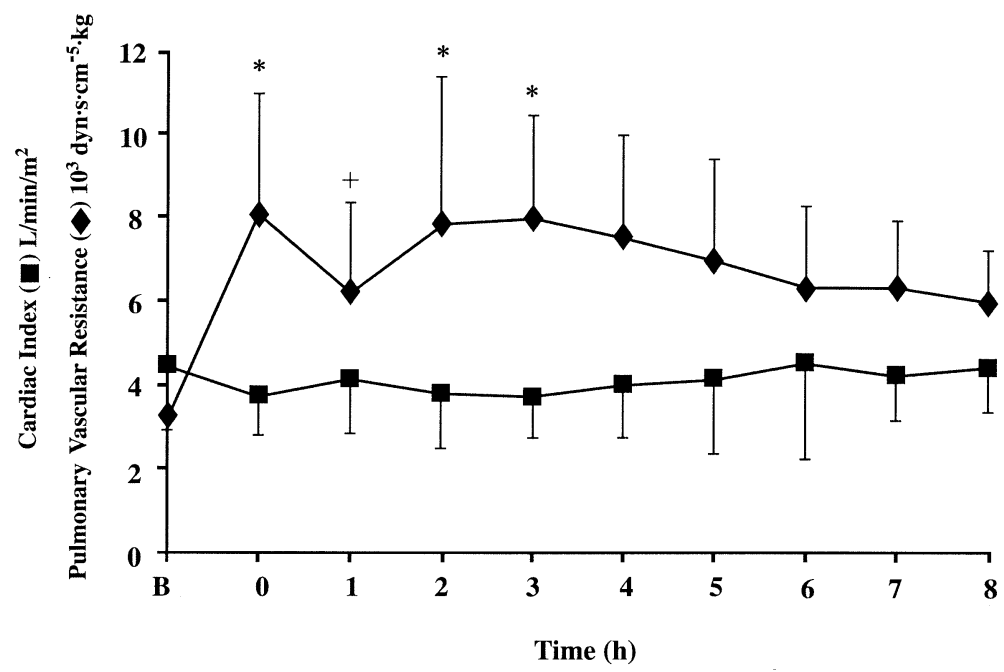

Figure 2. Cardiac index and pulmonary vascular resistance versus time ( $B$, baseline). Time 0 indicates the commencement of cavopulmonary assist. Cardiac index remained statistically unchanged throughout the study. Pulmonary vascular resistance, although initially increased, trended downward and was significantly lower at time 8 compared with times 2 and 3 . All cavopulmonary assist time points were significantly different from baseline $(P \leq .003)$. *Significant difference from the 8-hour time point $(P \leq .039)$; + significant difference from time $0(P=$ .043). Error bars represent SD.

TABLE 1. Hemodynamic and gas-exchange data with newborn cavopulmonary assist

\begin{tabular}{|c|c|c|c|c|}
\hline Parameter & Baseline & $\begin{array}{c}1-\mathrm{h} \\
\text { cavopulmonary } \\
\text { assist }\end{array}$ & $\begin{array}{c}\text { 8-h } \\
\text { cavopulmonary } \\
\text { assist }\end{array}$ & $P$ value \\
\hline Heart rate (bpm) & $188 \pm 26$ & $189 \pm 37$ & $172 \pm 34$ & \\
\hline Systolic blood pressure & $73 \pm 12$ & $70 \pm 15$ & $72 \pm 16$ & \\
\hline Diastolic blood pressure & $41 \pm 10$ & $32 \pm 10$ & $26 \pm 7 \dagger$ & .002 \\
\hline Pulmonary artery pressure & $13.1 \pm 2.1$ & $21.2 \pm 3.1^{*}$ & $23.3 \pm 4.3^{*}$ & $<.001$ \\
\hline Left atrial pressure & $3.5 \pm 1.8$ & $4.2 \pm 1.3$ & $5.2 \pm 1.5^{*}$ & .025 \\
\hline Inferior vena caval pressure & $4.3 \pm 1.8$ & $5.2 \pm 1.3$ & $7.8 \pm 2.4^{*}$ & $<.001$ \\
\hline $\operatorname{MCFP}(n=5)$ & $8 \pm 1.8$ & & $11 \pm 2.4^{*}$ & .021 \\
\hline Cardiac index & $4.45 \pm 1.53$ & $4.14 \pm 1.33$ & $4.38 \pm 1.04$ & \\
\hline Systemic blood flow & $253.9 \pm 84.0$ & $238.9 \pm 86.8$ & $251.8 \pm 69.0$ & \\
\hline Pulmonary vascular resistance & $3.24 \pm 1.09$ & $6.22 \pm 2.13^{*}$ & $5.94 \pm 1.29^{*}$ & $<.001$ \\
\hline Lactate $(\mathrm{mmol} / \mathrm{L})$ & $3.06 \pm 1.24$ & $3.98 \pm 2.26$ & $5.88 \pm 4.14$ & \\
\hline Hemoglobin $(\mathrm{g} / \mathrm{dL})$ & $9.7 \pm 2.4$ & $9.2 \pm 2.1$ & $7.4 \pm 1.6 \dagger$ & .019 \\
\hline $\mathrm{pH}$ & $7.47 \pm 0.15$ & $7.48 \pm 0.07$ & $7.38 \pm 0.07 \dagger$ & .043 \\
\hline $\mathrm{PCO}_{2}(\mathrm{~mm} \mathrm{Hg})$ & $32.9 \pm 9.7$ & $28.4 \pm 6.1$ & $35.3 \pm 5.7$ & \\
\hline $\mathrm{PO}_{2}(\mathrm{~mm} \mathrm{Hg})$ & $442.8 \pm 91.4$ & $449.8 \pm 125.2$ & $447.4 \pm 124.4$ & \\
\hline $\mathrm{HCO}_{3}{ }^{-}(\mathrm{mmol} / \mathrm{L})$ & $24 \pm 4$ & $21 \pm 4$ & $20 \pm 3^{*}$ & .038 \\
\hline Base excess (mmol/L) & $0.8 \pm 4.6$ & $-1.4 \pm 3.9$ & $-3.8 \pm 3.3^{*}$ & .020 \\
\hline
\end{tabular}

Baseline refers to conditions before cavopulmonary diversion. Cavopulmonary assist data are shown at 1- and 8-hour periods after initiation of cavopulmonary assist. Values are expressed as mean \pm SD in 13 lambs. Pressures are in $\mathrm{mm} \mathrm{Hg}$. Cardiac index is expressed as $\mathrm{L} / \mathrm{min} / \mathrm{m}^{2}$. Systemic blood flow is calculated as cardiac output/weight and is expressed as $\mathrm{mL} \cdot \mathrm{min}^{-1} \cdot \mathrm{kg}^{-1}$. Pulmonary vascular resistance is calculated as $\left(\mathrm{P}_{\mathrm{PA}}-\mathrm{P}_{\mathrm{LA}}\right) / \mathrm{SBF}$ and expressed as $10^{3} \mathrm{dyn} \cdot \mathrm{s} \cdot \mathrm{cm}^{-5} \cdot \mathrm{kg}^{-1}$. MCFP, Mean circulatory filling pressure; $S B F$, systemic blood flow; PPA, mean pulmonary artery pressure; $P L A$, mean left atrial pressure.

*Significantly different from baseline, tsignificantly different from baseline and the 1-hour cavopulmonary assist period.

Neither pulmonary edema nor hemorrhage was observed. High partial pressures of oxygen were maintained without acute compromise in circulatory stability.

\section{Discussion}

This study demonstrates relative hemodynamic stability in a neonatal animal model of pump-assisted cavopulmonary 
blood flow when compared with prior similar models of univentricular circulation in which cavopulmonary support was not applied. ${ }^{12,13}$ Heart rate, systolic blood pressure, and cardiac index remained at baseline levels. The significant decrease in diastolic blood pressure and increase in left atrial pressure, a reflection of diminished left ventricular function, are attributable to extensive surgical intervention, vasoactive and myocardial depressive effects of chronic inhaled anesthetic, and withholding inotropic support. Slight increases in inferior vena caval pressure and mean circulatory filling pressure are consistent with mild volume loading. The systemic venous pressure increases in this study were minimal compared with previous experimental models of Fontan circulation without mechanical assistance, in which a central venous pressure of more than 20 to 25 $\mathrm{mm} \mathrm{Hg}$ was required to overcome the cavopulmonary pressure gradient and provide adequate preload to the systemic ventricle.

Arterial lactate, base excess, and $\mathrm{pH}$ measurements indicate a trend toward metabolic acidosis over the course of the study. This suggests suboptimal systemic perfusion, but it did not affect overall hemodynamic stability. Baseline cardiac output in this model likely underestimates basal cardiac output in awake animals due to myocardial depressant effects of inhaled anesthetic. Because cavopulmonary assist pump output was matched to baseline cardiac output in the cavopulmonary assist period, this possibly contributed to inadequate systemic perfusion. The addition of inotropic support to maintain ventricular function could alleviate the problem of inadequate systemic perfusion.

Pulmonary artery pressures and pulmonary vascular resistance increased significantly from baseline after pump flow was instituted. This was not unexpected given the high pulmonary vascular reactivity of the neonate, the presence of mild metabolic acidosis, and the continuous nature of pulmonary blood flow in contrast to physiologic pulsatile flow. ${ }^{14,15}$ Actual increases in pulmonary artery pressures were modest $(<30 \%$ systemic pressure), and no extended periods of pulmonary hypertension $(>50 \%$ systemic pressure) were observed. Furthermore, pulmonary vascular resistance decreased significantly through the last 6 hours of the study. The use of pulmonary vasodilators could lessen absolute pulmonary pressures to more closely approximate baseline.

An assumption that a reactive newborn pulmonary vasculature will yield a transpulmonary gradient prohibitive to Fontan circulation may be inaccurate if ideal cavopulmonary flow conditions are provided. In healthy neonates, pulmonary vascular resistance decreases precipitously to within $10 \%$ of adult baseline values within hours after birth and is maintained at these low levels despite the presence of the reactive transitional pulmonary substrate. ${ }^{16}$ The paradox of the Fontan circulation is that it produces simultaneous vena caval hypertension and pulmonary arterial hypotension. ${ }^{17}$ Theoretically, minimal hydraulic support would be required to overcome the 10 to $20 \mathrm{~mm} \mathrm{Hg}$ cavopulmonary pressure gradient needed to augment flow from the systemic venous circulation to the pulmonary circulation and reverse the paradox. Implantable devices that have been developed for systemic circulatory support are capable of performing this task and may make it possible to overcome the potentially increased pulmonary vascular resistance of the neonate.

There are several compelling theoretical advantages underlying the rationale for cavopulmonary assist (Figure 3). Most importantly, the univentricular circulation is restored to one resembling 2-ventricle physiology with a series arrangement of the pulmonary and systemic circulations. The single ventricle is not subjected to volume overload and must pump only 1 ventricular output while a device supports the equivalent of right ventricular output. An early reduction of volume work has demonstrated benefit to longterm myocardial function for single-ventricle physiology. ${ }^{18,19}$ Coronary perfusion pressure, which is dependent on diastolic blood pressure, could theoretically be preserved. ${ }^{8}$ Partial pressure of oxygen can be maintained within the physiologically normal range without inducing circulatory balance instability, as seen in classic Norwood physiology. Fully oxygenated blood $\left(\mathrm{PO}_{2} \geq 80 \mathrm{~mm} \mathrm{Hg}\right)$ improves myocardial performance, optimizes function of highly oxygendependent organ systems (eg, brain and kidney), and minimizes pulmonary vascular resistance, thus indirectly reducing the circulatory support requirement. Finally, the transitional pulmonary vasculature is not exposed to hypoxemia, and this prevents pathologic pulmonary vascular remodeling. ${ }^{6}$

This work introduces several novel physiologic concepts. The first is the effect of cavopulmonary assist on chronic maturation of the newborn transitional pulmonary vasculature. The second is the response of the newborn systemic venous circulation to a gradual transition to Glenn or Fontan physiology. The third is technical issues related to application of this type of circulatory support in a neonate, including duration of support, potential benefit of pulsatile perfusion, risk of thrombosis, and growth potential of surgical pathways.

This work is highly theoretical, and any application to the clinical arena remains speculative. The concept could be applied either to partial (Glenn) cavopulmonary diversion or to total (Fontan) cavopulmonary diversion. The study is limited by the absence of true single-ventricle anatomy, and the functional ventricle is a morphologically left ventricle. One hundred percent inspired oxygen was used to show circulatory stability at high blood oxygen concentrations; this is not observed in stage 1 Norwood physiology. The decrease in diastolic blood pressure cannot be attributed to 
A

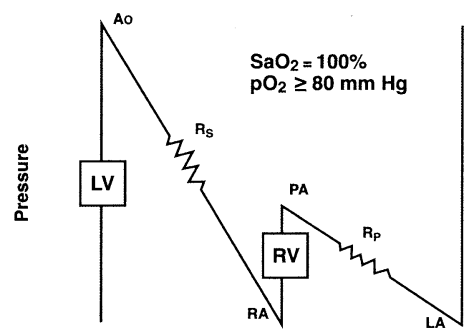

B

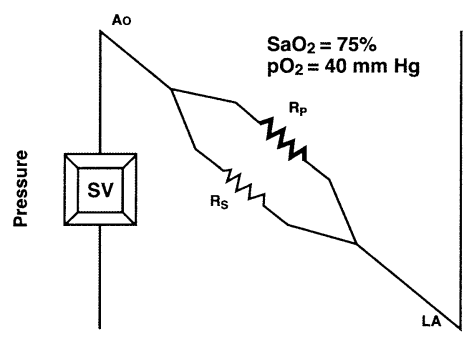

C

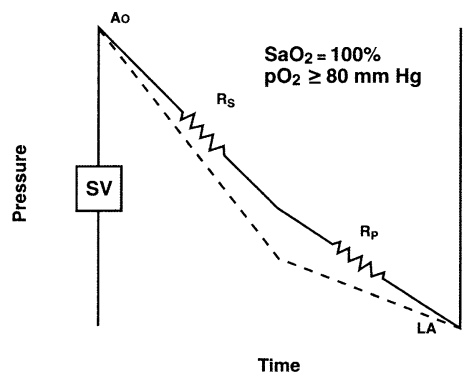

Cavopulmonary Assist

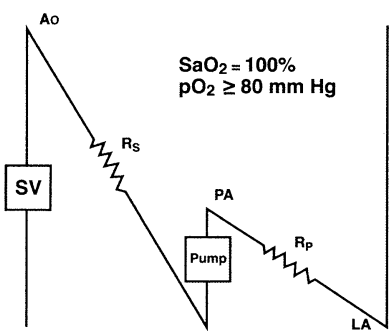

E Weaning Cavopulmonary Assist

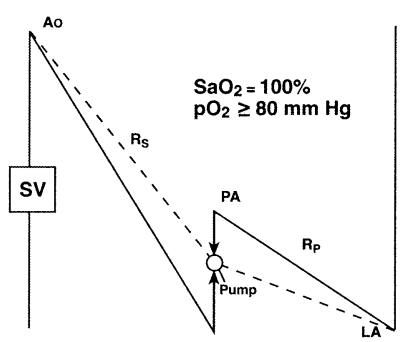

$\mathbf{F}$

Off Cavopulmonary Assist

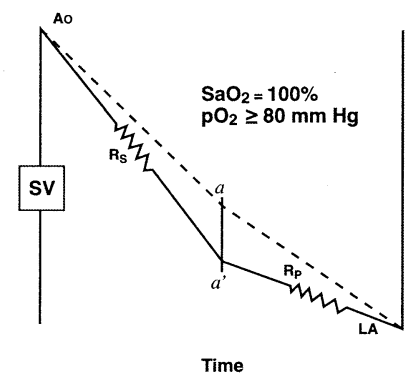

Figure 3. Theoretical circulatory energetics of cavopulmonary assist. Pressure-time diagrams schematically illustrate the work function of various physiologic states. A, Normal circulation. B, Norwood circulation. Of note are a parallel arrangement of the systemic and pulmonary circulations, increased pulmonary vascular resistance, and volume load on the single ventricle. C, Fontan circulation. The systemic and pulmonary circulations are in series. D, Cavopulmonary assist; replicates 2-ventricle physiology (in comparison to A). E, The circulation as cavopulmonary assist is being weaned. With decreasing support, systemic venous pressure increases and pulmonary arterial pressure decreases to converge at equilibrium. Pulmonary vascular resistance is a principal determinant of the equilibrium point, and a lower value portends more favorable Fontan hemodynamics. $F$, The circulation after cavopulmonary assist has been weaned. This is similar to the Fontan circulation shown in C. The dashed line represents postulated increased work resulting from pulmonary vascular maladaptation following the classic staged surgical approach. The area between the 2 curves, denoted by a-a', represents a theoretical work-reduction benefit to the single ventricle, assuming that the pulmonary vasculature matures more normally under conditions of cavopulmonary assist. $L V$, Left ventricle; $R V$, right ventricle; $S V$, single ventricle; $A o$, aorta; $P A$, pulmonary artery; $L A$, left atrium; $R_{s}$, systemic vascular resistance; $R_{p}$, pulmonary vascular resistance.

an anatomic cause, however, and, on the basis of our results, does not seem to be due to inadequate preload to the single ventricle or to low cardiac output. This is a serious limitation that seems to be related to the anesthetic regimen, a conservative fluid management strategy, and a lack of inotropic support in an intricate neonatal model of univentricular circulation. The steady flow conditions provided by the pump may have had detrimental effects on the pulmonary vasculature in comparison to pulsatile flow, and this will require additional study. Despite these limitations, this study demonstrates the feasibility of cavopulmonary assist in maintaining relative stability in an acute experimental model of newborn total cavopulmonary diversion and univentricular circulation. 


\section{References}

1. Ashburn DA, McCrindle BW, Tchervenkov CI, Jacobs ML, Lofland GK, Bove EL, et al. Outcomes after the Norwood operation in neonates with critical aortic valve stenosis or aortic valve atresia. $J$ Thorac Cardiovasc Surg. 2003;125:1070-82.

2. Mahle WT, Spray TL, Wernovsky G, Gaynor JW, Clark BJ. Survival after reconstructive surgery for hypoplastic left heart syndrome. A 15-year experience from a single institution. Circulation. 2000; 102(suppl 3):III136-41.

3. Mahle WT, Spray TL, Gaynor JW, Clark BJ. Unexpected death after reconstructive surgery for hypoplastic left heart syndrome. Ann Thorac Surg. 2001;71:61-5.

4. Fenton KN, Siewers RD, Rebovich B, Pigula FA. Interim mortality in infants with systemic-to-pulmonary shunts. Ann Thorac Surg. 2003; 76:152-7.

5. Wernovsky G, Newburger J. Neurologic and developmental morbidity in children with complex congenital heart disease. J Pediatr. 2003; 142:6-8.

6. Rudolph AM. Congenital diseases of the heart: clinical-physiological considerations. 2nd ed. Armonk (NY): Futura; 2001. p. 142.

7. Bartram U, Grunenfelder J, Van Praagh RV. Causes of death after the modified Norwood procedure: a study of 122 postmortem cases. Ann Thorac Surg. 1997;64:1795-802.

8. Fogel MA, Rychik J, Vetter J, Donofrio MT, Jacobs ML. Effect of volume unloading surgery on coronary flow dynamics in patients with aortic atresia. J Thorac Cardiovasc Surg. 1997;113:718-27.

9. Sano S, Ishino K, Kawada M, Arai S, Kasahara S, Asai T, et al. Right ventricle-pulmonary artery shunt in first-stage palliation of hypoplastic left heart syndrome. J Thorac Cardiovasc Surg. 2003;126:504-10.

10. Reddy VM, Liddicoat JR, Hanley FL. Primary bidirectional superior cavopulmonary shunt in infants between 1 and 4 months of age. Ann Thorac Surg. 1995;59:1120-6.

11. Rothe CF. Mean circulatory filling pressure: its meaning and measurements. J Appl Physiol. 1993;74:499-509.

12. Mace L, Dervanian P, Bourriez A, Mazmanian GM, Lambert V, Losay $\mathrm{J}$, et al. Changes in venous return parameters associated with univentricular Fontan circulations. Am J Physiol Heart Circ Physiol. 2000; 279:H2335-43.

13. Kaku K, Matsuda H, Kaneko M, Matsumura R, Shirakura R, Nakano $\mathrm{S}$, et al. Experimental complete right heart bypass. Proposal of a new model and acute hemodynamic assessment with vasoactive drugs in dogs. J Thorac Cardiovasc Surg. 1990;99:161-6.

14. Johnson EH, Bennett SH, Goetzman BW. The influence of pulsatile perfusion on the vascular properties of the newborn lamb lung. Pediatr Res. 1992;31:349-53.

15. Presson RG, Baumgartner WA, Peterson AJ, Glenny RW, Wagner WW. Pulmonary capillaries are recruited during pulsatile flow. J Appl Physiol. 2002;92:1183-90.

16. Rudolph AM, Auld PA, Galinko RJ, Paul MH. Pulmonary vascular adjustments in the neonatal period. Pediatrics. 1961;55:28-34.

17. de Leval MR. The Fontan circulation: what have we learned? What to expect? Pediatr Cardiol. 1998;19:316-20.

18. Mahle WT, Wernovsky G, Bridges ND, Linton AB, Paridon SM. Impact of early ventricular unloading on exercise performance in preadolescents with single ventricle Fontan physiology. J Am Coll Cardiol. 1999;34:1637-43.

19. Ohuchi H, Yasuda K, Hasegawa S, Miyazaki A, Takamuro M, Yamada $\mathrm{O}$, et al. Influence of ventricular morphology on aerobic exercise capacity in patients after the Fontan operation. J Am Coll Cardiol. 2001;37:1967-74.

\section{Targeted}

The Journal of Thoracic and Cardiovascular Surgery gives you two tables of contents.

The condensed table of contents tells you at a glance what topics and authors are presented each month. The expanded table of contents gives you a brief abstract of each article. You select only those articles of most interest to you for further reading. 